\title{
Deep Learning-Based Strategy For Macromolecules Classification with Imbalanced Data from Cellular Electron Cryotomography
}

\author{
$3^{\text {rd }}$ Zhipeng Bao \\ Department of Electronic Engineering \\ Tsinghua University \\ Beijing, China \\ bzp15@mails.tsinghua.edu.cn
}

$1^{\text {st }}$ Ziqian Luo

International School

Beijing University of Posts and Telecommunications

Beijing, China

luoziqian@bupt.edu.cn

\author{
$2^{\text {nd }}$ Xiangrui Zeng \\ Computational Biology Department \\ Carnegie Mellon University \\ Pittsburgh, United States \\ xiangruz@andrew.cmu.edu
}

\author{
$4^{\text {th }} \operatorname{Min} \mathrm{Xu}$ \\ Computational Biology Department \\ Carnegie Mellon University \\ Pittsburgh, United States \\ mxu1@cs.cmu.edu
}

\begin{abstract}
Deep learning model trained by imbalanced data may not work satisfactorily since it could be determined by major classes and thus may ignore the classes with small amount of data. In this paper, we apply deep learning based imbalanced data classification for the first time to cellular macromolecular complexes captured by Cryo-electron tomography (Cryo-ET). We adopt a range of strategies to cope with imbalanced data, including data sampling, bagging, boosting, Genetic Programming based method and. Particularly, inspired from Inception 3D network, we propose a multi-path CNN model combining focal loss and mixup on the Cryo-ET dataset to expand the dataset, where each path had its best performance corresponding to each type of data and let the network learn the combinations of the paths to improve the classification performance. In addition, extensive experiments have been conducted to show our proposed method is flexible enough to cope with different number of classes by adjusting the number of paths in our multi-path model. To our knowledge, this work is the first application of deep learning methods of dealing with imbalanced data to the internal tissue classification of cell macromolecular complexes, which opened up a new path for cell classification in the field of computational biology.
\end{abstract}

\section{INTRODUCTION}

Biological pathways depend on the function of macromolecular complexes, whose structure and spatial organization are critical to the function and dysfunction of pathways. Due to the limitations of data acquisition techniques, the native structural information of macromolecular complexes is extremely difficult to obtain [1]. With the development of biotechnology, Cryo-electron tomography (Cryo-ET) enables 3D visualization of cellular tissue in near-native state and sub-molecular resolution [2]-[4], making it a powerful tool for analyzing macromolecular complexes and their spatial organization within single cells [5].

Corresponding author: $\mathrm{Min} \mathrm{Xu}$
However, it is often observed that the macromolecular complex data collected is imbalanced. The protein concentration difference can be as large as seven orders of magnitude [6]. One type of macromolecular complexes may dominate over other types, resulting in a low accuracy. In fact, the problem of data imbalance also occurs in most real-world classification problems. The collected data follows a long tail distribution i.e., data for few object classes is abundant while data for others is scarce. This phenomenon is termed the data-imbalanced classification problem [7]. Although the problem of data-imbalanced classification occurs frequently in the computer vision field, research work on this topic has been rare in recent years. Almost all competitive datasets avoid data-imbalanced during the evaluation and training procedures. For instance, the case of the popular image classification datasets (such as CIFAR10/100, ImageNet, Caltech101/256, and MIT67), efforts have been made by the collectors to ensure that, either all of the classes have a minimum representation with sufficient data, or that the experimental protocols are reshaped to use an equal number of images for all classes during the training and testing processes [8], [9].

In this paper, we conduct extensive experiments and explore various methods for dealing with the problem of dataimbalanced classification, such as data sampling, bagging, boosting, Genetic Programming based method. We rigorously prove that the above various methods are indeed effective, and apply them to the classification of macromolecular complexes in single cells, and achieved a competitive classification performance. In particular, we make the following key contributions:

- We summarize various well-known methods for dealing with data-imbalanced classification problems in order to improve classification performance and further find 
the best combinations with our own model among the methods.

- We apply the method of dealing with imbalanced data to the classification of cell macromolecular complexes for the first time.

- We propose a novel method to solve the data-imbalanced problem, termed as multi-path convolutional neural network (CNN), which significantly improves the classification performance over traditional methods. Moreover, our model is flexible enough to cope with different number of classes by adjusting the number of paths in our multi-path CNN model.

\section{RELATED WORK}

\section{A. Cryo-electron tomography (Cryo-ET)}

Cryo-electron tomography (Cryo-ET) is an imaging technique used to produce high-resolution $(4 \mathrm{~nm})$ threedimensional views of samples, typically biological macromolecules in cells [9]. Cryo-ET is a specialized application of transmission electron cryomicroscopy (CryoTEM) in which samples are imaged as they are tilted, resulting in a series of $2 \mathrm{D}$ images that can be combined to produce a $3 \mathrm{D}$ reconstruction, similar to a CT scan of the human body. In contrast to other electron tomography techniques, samples are immobilized in non-crystalline ("vitreous") ice and imaged under cryogenic conditions $\left(<150^{\circ} \mathrm{C}\right)$, allowing them to be imaged without dehydration or chemical fixation, which could otherwise disrupt or distort biological structures [9], [10]. Cryo-electron tomography (Cryo-ET) [11]-[13] enables the $3 \mathrm{D}$ visualization of structures at close-to-native state and in sub-molecular resolution within single cells $[14]-[17]$.

\section{B. Inception3D Network}

[18] propose a 3D variant of tailored inception network [19], denoted as Inception3D. Inception network is a recent successful CNN architecture that has the ability to achieve competitive performance with relatively low computational cost [18]. CNN [17] are well-known for extracting features from a image by using convolutional kernels and pooling layers to emulates the response of an individual to visual stimuli. This work [20] is the first application of deep learning for systematic structural discovery of macromolecular complexes among large amount (millions) of structurally highly heterogeneous particles captured by Cryo-ET. It represents an important step towards large scale systematic detection of native structures and spatial organizations of large macromolecular complexes inside single cells.

\section{Mixup: Data-Dependent Data Augmentation}

Large deep neural networks are powerful, but exhibit undesirable behaviors such as memorization and sensitivity to adversarial examples. [21] propose mixup, a simple learning principle to alleviate these issues. Essentially, mixup trains a neural network on convex combinations of pairs of examples and their labels. By doing so, mixup regularizes the neural network to favor simple linear behavior in-between training examples, and here is how the mixup training loss is defined:

$\mathcal{L}(\theta)=\mathbb{E}_{x_{1}, y_{1} \sim p_{\text {train }}} \mathbb{E}_{x_{2}, y_{2} \sim p_{\text {train }}} \mathbb{E}_{\lambda \sim \beta(0.1)} \ell\left(\lambda x_{1}+(1-\right.$ 入) $\left.x_{2}, \lambda y_{1}+(1-\lambda) y_{2}\right)$

\section{Focal loss}

[22], [49] discover that the extreme foreground-background class imbalance encountered during training of dense detectors is the central cause. We propose to address this class imbalance by reshaping the standard cross entropy loss such that it downweights the loss assigned to well-classified examples. [23] proposed a novel Focal Loss focuses training on a sparse set of hard examples and prevents the vast number of easy negatives from overwhelming the detector during training.

\section{E. Sampling}

1) Oversampling: Oversampling method achieves sample balanced by increasing the number of minority samples in the classification. The most straightforward way is to simply copy a few samples to form multiple records. However, the disadvantage of this method is that if there are few sample features, it may lead to over-fitting problems. Improved oversampling method by adding random noise, interference data, or certain rules to generate new synthetic samples in a few classes, such as SMOTE algorithm. The process is described about SMOTE method in Algorithm 1.

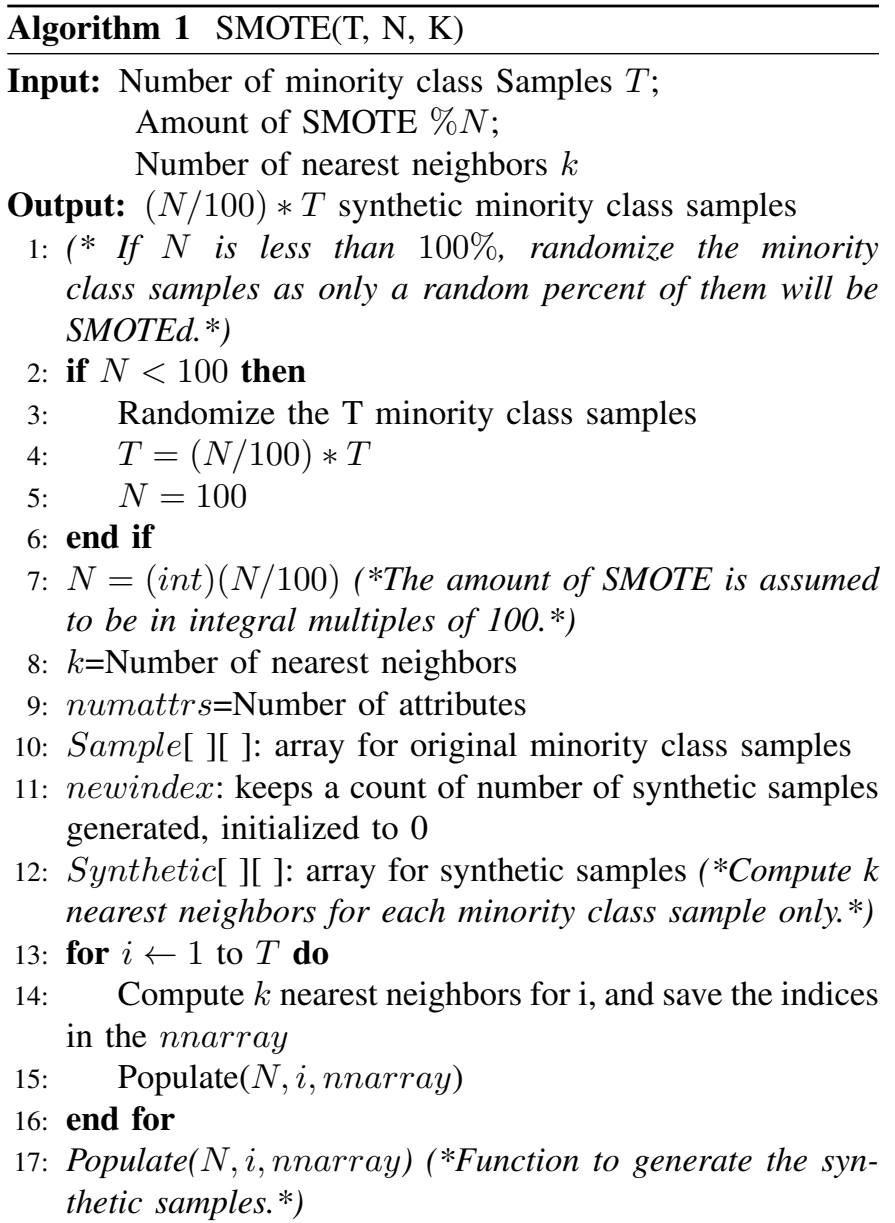




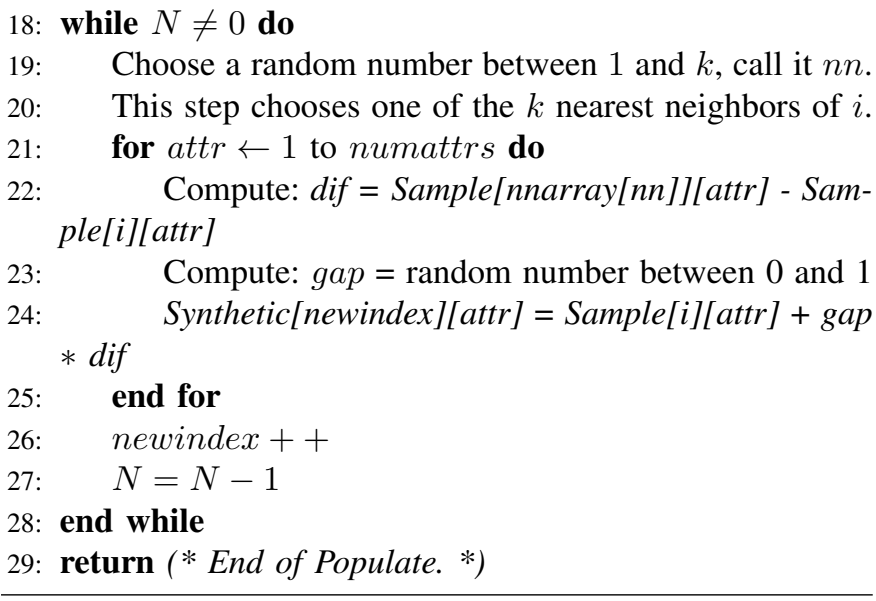

2) Undersampling: Another popular method [24], [46] that results in having the same number of examples in each class. However, as opposed to oversampling, examples are removed randomly from majority classes until all classes have the same number of examples. While it might not appear intuitive, there is some evidence that in some situations undersampling can be preferable to oversampling [25]. A significant disadvantage of this method is that it discards a portion of available data. To overcome this shortcoming, some modifications were introduced that more carefully select examples to be removed. E.g. one-sided selection identifies redundant examples close to the boundary between classes [26]. More general approach than undersampling is data decontamination that can involve relabeling of some examples [27].

\section{OUR METHOD}

\section{A. Overview of our model}

With the inspiration from Inception 3D network [28], [44], [45], we propose a novel model termed as multi-path $\mathrm{CNN}$, combaning with mixup and focal loss method, which could significantly improve performance on the imbalanced CryoET data. The overview of our proposed model is as shown in Figure 1.

\section{B. Mixup on the Cryo-ET dataset}

Motivated by [29], [42], [43], we introduce a simple and data-agnostic data augmentation routine, termed as mixup. In a nutshell, mixup constructs virtual training examples

$$
\begin{aligned}
& \tilde{x}=\lambda x_{i}+(1-\lambda) x_{j} \\
& \tilde{y}=\lambda y_{i}+(1-\lambda) y_{j}
\end{aligned}
$$

where $x_{i}, x_{j}$ are raw input vectors, and $y_{i}, y_{j}$ are one-hot label encodings $\left(x_{i}, y_{i}\right)$ and $\left(x_{j}, y_{j}\right)$ are two examples drawn at random from our training data, and $\lambda \in[0,1]$. Therefore, mixup extends the training distribution by incorporating the prior knowledge that linear interpolations of feature vectors should lead to linear interpolations of the associated targets.

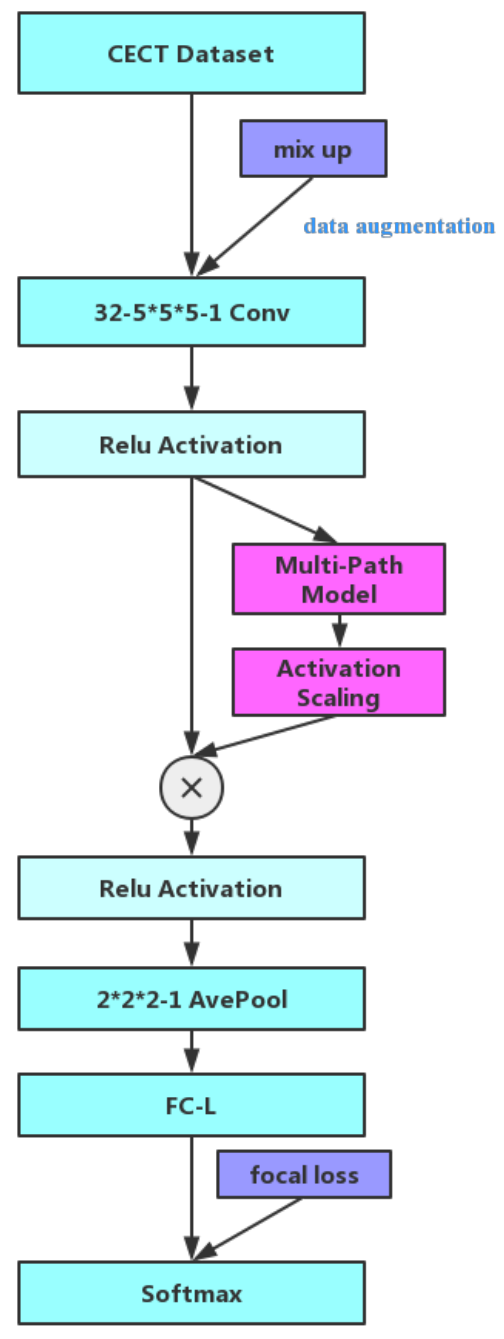

Fig. 1. Overview architectures of our model.

\section{Multi-path CNN}

Many subtomogram data from Cryo-ET are imbalanced due to their different ratio in the cell. However, there is little work that had been done to solve the problem of imbalanced data from Cryo-ET. In this section, we describe detaily our proposed multi-path CNN model showed in Figure 2, which will be useful to cope with the imbalanced data from Cryo-ET with the combination of the related works from Section 2.

Unlike traditional CNN which only has a single path with serial combinations of convolutional kernels and pooling layers, our multi-path $\mathrm{CNN}$ has multiple parallel combinations of convolutional kernels and pooling layers, based on the composition of our imbalanced data. More specifically, the number of classes from Cryo-ET equals to the number of parallel paths in our multi-path CNN model. Each path will try to learn from the imbalanced Cryo-ET data and become the best classifier corresponding to a certain type of data before the 


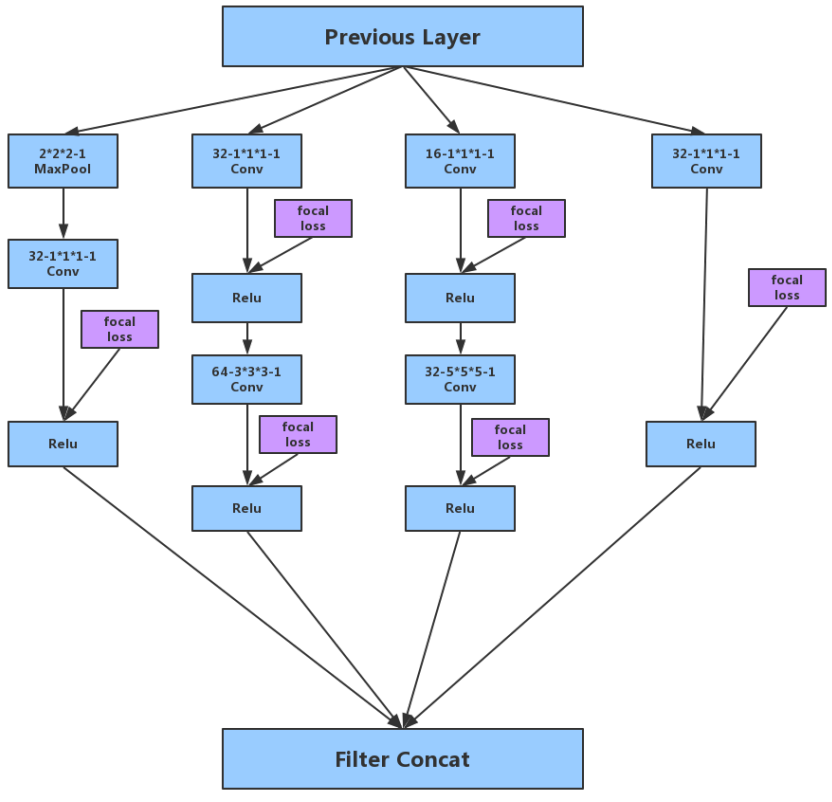

Fig. 2. Model of multi-path CNN

concatenate layer. The reason about firstly trying to determine each single path for each type of data from Cryo-ET is that it will be easier to find a classifier through deep learning that will behave well in recognizing a single class of data, regardless of other classes.

In order to find the best classifier for a certain type of data from Cryo-ET, we firstly carry out a lot of experiments with serial CNN and find its best structure to recognize a single type from the imbalanced dataset. All paths will be concatenated together when they are identified respectively. It is worth mentioning that sampling methods have not been used in the whole process since it may lead to overfitting or underfitting. Each single path learns how to do the classification job from the original imbalanced data and whole multi-path CNN learns how to balance between these paths and make a more precise decision towards all the types of data from Cryo-ET.

\section{Filter Concat}

The final model obtains the most suitable convolution kernel on each path, so that the model effect is optimal, and then combines the best parameters learned by the models on the four paths, and finally enters the new pooling layer through a filter. The final classification result is obtained by softmax through the full connection of the L layer.

\section{E. Focal Loss for imbalanced classification}

We use an $\alpha$-balanced variant of the focal loss:

$$
F L\left(p_{t}\right)=-\alpha_{t}\left(1-p_{t}\right)^{\gamma} \log \left(p_{t}\right)
$$

We adopt this form in our experiments as it yields slightly improved accuracy over the non- $\alpha$-balanced form. Finally, we note that the implementation of the loss layer combines the sigmoid operation for computing $\mathrm{p}$ with the loss computation, resulting in greater numerical stability.

\section{F. Evaluation metrics}

Evaluation metrics play an important role in assessing the classification performance and guiding the model design. Most of the traditional methods dealing with the imbalanced data concentrate on binary classification. In binary classification problem, class labels can be divided as positive and negative. As the confusion matrix shows in Table 1, true positive (TP) and true negative (TN) denote the number of positive and negative samples that are correctly classified while false negative (FN) and false positive (FP) denote the number of positive and negative samples that are wrongly classified.

Accuracy is the most commonly used metric to evaluate model performance, however, it is no longer a proper measure in imbalanced classification problem since the minor class has minimal impact on accuracy compared with the major class. To solve the problem, a pair of metrics, precision and recall, have been adopted.

$$
\begin{gathered}
\text { precision }=\frac{T P}{T P+F P} \\
\text { recall }=\frac{T P}{T P+F N}
\end{gathered}
$$

Meanwhile, F-Score is used to integrate precision and recall into a single metric for convenient evaluation of model.

$$
\mathrm{F}_{\beta}=\left(1+\beta^{2}\right) \cdot \frac{\text { precision } \cdot \text { recall }}{\left(\beta^{2} \cdot \text { precision }\right)+\text { recall }}
$$

Where $\beta$ represents the weight between precision and recall. During our evaluation process, we set $\beta=1$ since we regard precision and recall has the same weight thus $F_{1}$-score is adopted.

However, in multi-class classification, we use Macro $F_{1}$ Score to evaluate the result.

$$
\text { Macro } F_{1}=\frac{\sum_{n=1}^{N} F_{1 n}}{N}
$$

where $\mathrm{n}$ represents the number of classification and $F_{1 n}$ is the $F_{1}$ score on nth category.

G-mean is another recognized metric derived from confusion matrix.

$$
G-\text { mean }=\sqrt{\frac{T P}{T P+F N}+\frac{T N}{T N+F P}}
$$

In multi-class classification, we also use Macro G-mean to evaluate the result, measure the balanced performance of a learning model.

$$
\text { Macro } G-\text { mean }=\frac{\sum_{n=1}^{N} G_{n}}{N}
$$

where $\mathrm{n}$ represents the number of classification and $G_{n}$ is the G-mean on nth category. 


\begin{tabular}{l|c|c}
\hline & pridicted positives & predicted negatives \\
\hline Real positives & TP & FN \\
Real negatives & FP & TN \\
\hline \multicolumn{2}{c}{ TABLE I } \\
\multicolumn{2}{c}{ CONFUSION MATRIX FOR BINARY CLASSIFICATION }
\end{tabular}

\begin{tabular}{c|cccc}
\hline & proteasome_d & ribosome & TRiC & proteasome_s \\
\hline Number & 1043 & 80 & 125 & 386 \\
\hline Ratio(\%) & 63.481 & 4.869 & 7.608 & 23.494 \\
\hline \multicolumn{4}{c}{ CLASS RATIO OF CRYO-ET DATASET. }
\end{tabular}

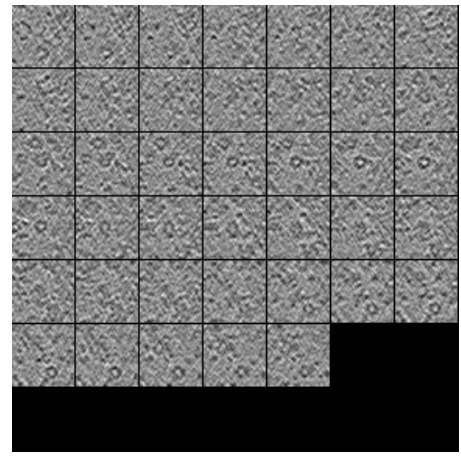

(a) proteasome_d

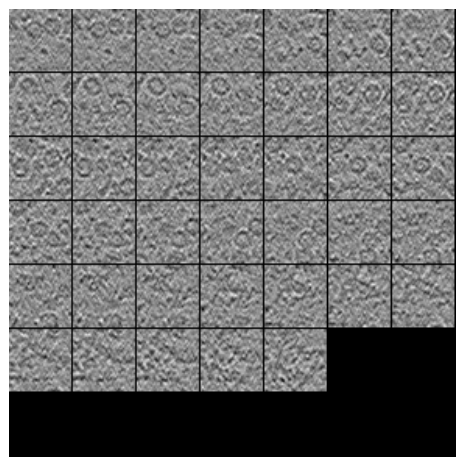

(c) TRiC

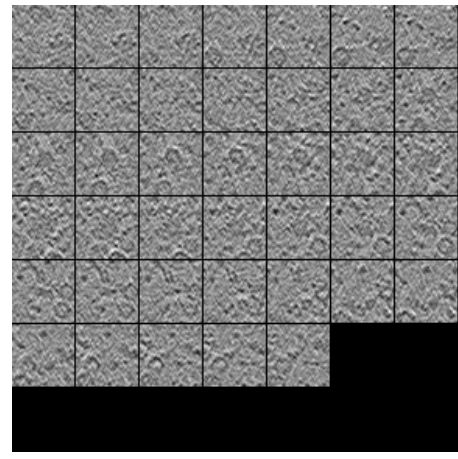

(b) ribosome

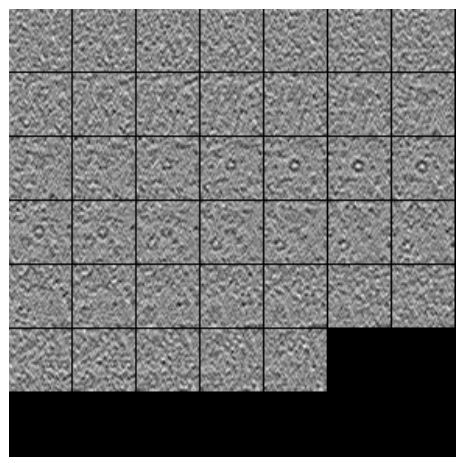

(d) proteasome_s

Fig. 3. The 2D visualizations of the $3 \mathrm{D}$ macro molecules

\section{EXPERIMENTS}

\section{A. Dataset details}

Furthermore, reference-free classification and averaging were tested on a dataset consisting of 125 TCP-1 ring complex (TRiC) subtomograms, 386 single capped proteasome (proteasome_s) subtomograms, 1043 double capped proteasome (proteasome_d) subtomograms, and 80 ribosome subtomograms extracted from a tomogram of rat neuron with expression of poly-GA aggregate. All subtomorgams were two times binned to size $40^{3}$ (voxel size: $1.368 \mathrm{~nm}$ ). The tilt angle range was $-50^{\circ}$ to $+70^{\circ}$.

The four types of macro molecules in our Cryo-ET dataset, which are proteasome_d, ribosome, TRiC, proteasome_s, whose imbalanced ratio are shown in Table 2. The Figure 3 are the $2 \mathrm{D}$ visualizations of the $3 \mathrm{D}$ macro molecules.
In order to train and test our multi-path CNN, we shuffle and split our dataset with two parts. There are 1307 samples in the training set and 327 samples in the testing set.

\section{B. Baseline Methods}

1) Bagging: [30] introduced the concept of bootstrap aggregating to construct ensembles. It consists in training different classifiers with bootstrapped replicas of the original training data-set. That is, a new data-set is formed to train each classifier by randomly drawing (with replacement) instances from the original data-set (usually, maintaining the original data-set size). Hence, diversity is obtained with the resampling procedure by the usage of different data subsets. Finally, when an unknown instance is presented to each individual classifier, a majority or weighted vote is used to infer the class [31].

2) Boosting: Boosting (also known as ARCing, adaptive resampling and combining) was introduced by Schapire in 


\begin{tabular}{c|cc|cc|cc|cc}
\hline \multirow{2}{*}{ Classes in Cryo-ET } & \multicolumn{2}{|c|}{ path 1 } & \multicolumn{2}{c|}{ path 2 } & \multicolumn{2}{c|}{ path 3 } & \multicolumn{2}{c}{ path 4 } \\
\cline { 2 - 9 } & F1 & G-mean & F1 & G-mean & F1 & G-mean & F1 & G-mean \\
\hline proteasome_d & $\mathbf{7 1 . 7}$ & $\mathbf{8 1 . 2}$ & 68.3 & 79.2 & 69.0 & 78.5 & 70.8 & 80.2 \\
ribosome & 69.7 & 78.1 & $\mathbf{7 4 . 2}$ & $\mathbf{8 2 . 6}$ & 72.3 & 79.4 & 71.4 & 77.6 \\
TRiC & 72.6 & 81.2 & 71.7 & 79.8 & $\mathbf{7 4 . 8}$ & $\mathbf{8 3 . 8}$ & 74.1 & 82.4 \\
proteasome_s & 71.2 & 80.4 & 69.2 & 78.5 & 72.7 & 81.9 & $\mathbf{7 3 . 3}$ & $\mathbf{8 3 . 0}$ \\
\hline
\end{tabular}

BINARY CLASSIFICATION BY FOUR DIFFERENT PATHS

\begin{tabular}{c|c|c}
\hline Model & \multicolumn{2}{|c}{ Cryo-ET } \\
\cline { 2 - 3 } Multi-path CNN & Macro F1 & Macro G-mean \\
\hline Multi-path CNN with boosting & 68.1 & 78.1 \\
Multi-path CNN with bagging & 69.3 & 79.3 \\
Multi-path CNN with SMOTE & 69.0 & 78.6 \\
Multi-path CNN with undersampling & 69.7 & 77.3 \\
Multi-path CNN with GP & 69.1 & 78.2 \\
Multi-path CNN with mixup & 70.4 & 80.2 \\
Multi-path CNN with focal loss & 70.9 & 80.7 \\
Multi-path CNN with SMOTE + boosting & 71.1 & 78.2 \\
Multi-path CNN with SMOTE + bagging & 70.1 & 80.2 \\
Multi-path CNN with SMOTE + GP & 71.4 & 81.5 \\
Multi-path CNN with mixup+focal loss & 72.6 & $\mathbf{8 4 . 7}$ \\
\hline
\end{tabular}

MULTI-PATH CNN COMBINED WITH DIFFERENT STRATEGIES ON IMBALANCED CRYO-ET

1990 [32]-[34]. Schapire proved that a weak learner (which is slightly better than random guessing) can be turned into a strong learner in the sense of probably approximately correct (PAC) learning framework. AdaBoost [35] is the most representative algorithm in this family, it was the first applicable approach of Boosting, and it has been appointed as one of the top ten data mining algorithms [36].

3) Genetic Programming (GP): GP [37] is an evolutionary algorithm technique inspired from biological evolution to find computer programs that perform a user-defined task, which can evolve biased classifiers when data sets are unbalanced. In GP, programs representing different solutions to a problem are combined with other programs to create new hopefully better programs; this process is repeated over a number of generations until a good solution is evolved [38]-[40]. [41] proposed GP methods utilize the unbalanced data as is in the learning phase, requiring no prior knowledge about the problem domain, to evolve classifiers with good classification ability on both minority and majority classes.

\section{Identification of each path in Multi-path CNN}

Before carrying out the experiment corresponding to the whole model in Figure 2, we have carried out lots of experiments to identify each suitable path in the multi-path CNN model. We name the four paths with path 1 , path 2 , path 3 and path 4, from left to right in the model in Figure 2. Each path has the best binary classification result on one of the four classes in Cryo-ET. For example, as shown in Table 3, path 1 behaves best on proteasome_d, while path 3 has the best result on TRiC class. During the experiment in each single path, we degenerate the multi-class classification problem with binary classification, say, the CNN branch in path 1 will only tell whether the input belongs to proteasome_d class and path 3 will only judge whether the input belongs to TRiC class.

\section{Multi-path CNN with traditional strategies on imbalanced dataset}

We have done some experiments to combine the multi-path CNN with recognized and effective strategies like boosting, bagging, SMOTE, focal loss and mixup method, towards the imbalanced dataset. The results are shown in Table 4.

Experiments have also been done to compare the multipath CNN with traditional classifier, Granular Support Vector Machine with Repetitive Undersampling(GSVM-RU), [47], [48]. Besides SVM modeling, GSVM-RU adds another hyperparameter $\mathrm{G}$, the number of negative granules. To solve the problems in multi-class classification with binary classifier, we use the method in Figure 4 to decompose the multi-class problem into a binary class problem. The experiment results are shown in Table 5.

Several pairs of experiments have also been done to show that our model be adjusted to two or three classes classification problem, rather than restricted in four classes classification problem. We conduct GSVM-RU on different number of classes as baseline for comparison. The results are shown in the Table 6 .

\section{E. Discussion}

Cryo-ET has become a powerful tool for 3D visualization of cellular components in sub-molecular resolution and nearprimary ecology [50]. However, imbalanced classification in cellular tomograms is difficult due to the high complexity of image content and imaging limitations. In order to complement the existing method, in this paper, we propose a multi-path CNN combined with mixup and focal loss strategy which will have the best classification result on the imbalanced data from Cryo-ET. The above experiment results demonstrate the power of our approach and they have also indicated that by changing the number of paths in our multi-path, the model can be 


\begin{tabular}{c|c|c}
\hline \multirow{2}{*}{ Model } & \multicolumn{2}{|c}{ Cryo-ET } \\
\cline { 2 - 3 } & Macro F1 & Macro G-mean \\
\hline Multi-path CNN with mixup + focal loss & $\mathbf{7 3 . 6}$ & $\mathbf{8 4 . 7}$ \\
\hline GSVM-RU TABLE V & 69.5 & 80.3 \\
\hline
\end{tabular}

COMPARISON BETWEEN MULTI-PATH CNN MODEL AND TRADITIONAL METHOD

\begin{tabular}{c|c|c}
\hline \multirow{2}{*}{ Model } & \multicolumn{2}{|c}{ Cryo-ET } \\
\cline { 2 - 3 } & Macro F1 & Macro G-mean \\
\hline Four-path CNN with mixup + focal loss & $\mathbf{7 3 . 6}$ & $\mathbf{8 4 . 7}$ \\
GSVM-RU(based on four classes) & 69.5 & 80.3 \\
\hline Three-path CNN with mixup + focal loss & 74.3 & 85.5 \\
GSVM-RU(based on three classes) & 70.1 & 81.6 \\
\hline Two-path CNN with mixup + focal loss & 76.4 & 87.2 \\
GSVM-RU(based on two classes) & 71.2 & 81.9 \\
\hline \multicolumn{2}{c}{ TABLE VI }
\end{tabular}

COMPARISON OF DIFFERENT NUMBER OF PATHS IN MULTI-PATH CNN

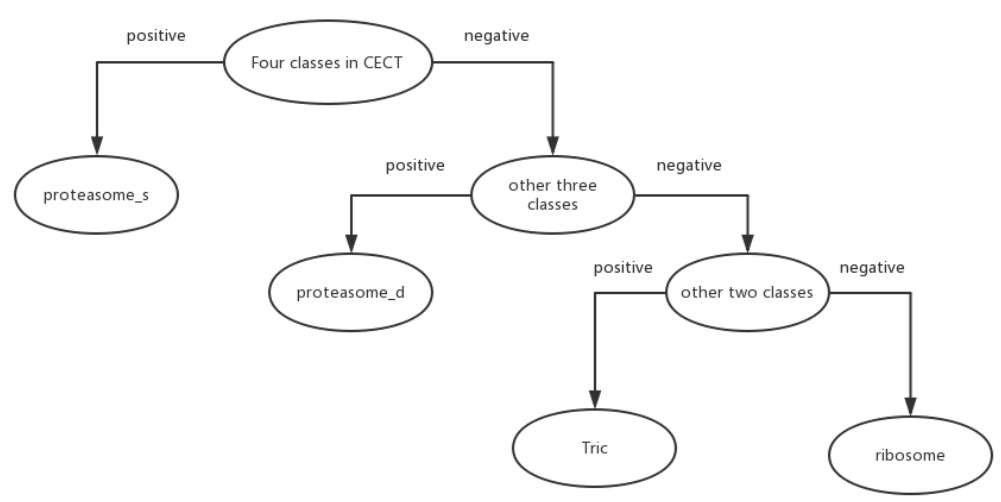

Fig. 4. Multiclass classification achieved by binary classifier

adapted to cope with imbalanced classification problems with different number of classes. The work provides useful steps for imbalanced classification in cell tomography. To the best of our knowledge, our work is the first application of CNNbased network with focal loss and mixup method in Cryo-ET data analysis. Our approach is a useful complement to current technology

\section{CONCLUSION}

In this paper, we apply the method of dealing with imbalanced data to the classification of cell macromolecular complexes for the first time, which opened up a new path for cell classification in the field of computational biology. In order to solve the imbalanced data problem from CryoET, we propose a multi-path CNN model combined with recognized strategies to deal with data imbalance issue like sampling, bagging and boosting and genetic programming. We have also made combinations among the methods and with our model. The multi-path CNN model consists of several independent paths that behave best in each class respectively. By adjusting the number of the paths in the model, we can deal with a more generalized classification problem with different number of classes. Experiments and comparisons with traditional classifiers have shown that the model can work effectively on the imbalanced data from Cryo-ET. In the future, we will also consider more issues in the field of computer and bio-related technologies to promote the development of computational biology.

\section{ACKNOWLEDGEMENT}

This work was supported in part by U.S. National Institutes of Health (NIH) grant P41 GM103712.

\section{REFERENCES}

[1] Xu M, Chai X, Muthakana H, et al. Deep learning-based subdivision approach for large scale macromolecules structure recovery from electron cryo tomograms[J]. Bioinformatics, 2017, 33(14): i13-i22.

[2] Zeng X, Leung M, Zeev-Ben-Mordehai T, Xu M. A convolutional autoencoder approach for mining features in cellular electron cryotomograms and weakly supervised coarse segmentation. Journal of Structural Biology (2017). arXiv:1706.04970

[3] Liu C, Zeng X, Wang K, et al. Multi-task Learning for Macromolecule Classification, Segmentation and Coarse Structural Recovery in CryoTomography[J]. arXiv preprint arXiv:1805.06332 2018.

[4] Wang K W, Zeng X, Liang X, et al. Image-derived generative modeling of pseudo-macromolecular structures-towards the statistical assessment of Electron CryoTomography template matching[J]. arXiv preprint arXiv:1805.04634. 2018.

[5] Zhao Y, Zeng X, Guo Q, et al. An integration of fast alignment and maximum-likelihood methods for electron subtomogram averaging and classification[J]. arXiv preprint arXiv:1804.01203 2018. 
[6] Beck M, Schmidt A, Malmstroem J, et al. The quantitative proteome of a human cell line. Molecular Systems Biology, volume 7, pages 549; November, 2011.

[7] Khan S H, Hayat M, Bennamoun M, et al. Cost-sensitive learning of deep feature representations from imbalanced data[J]. IEEE transactions on neural networks and learning systems, 2018, 29(8): 3573-3587.

[8] Lee C Y, Xie S, Gallagher P, et al. Deeply-supervised nets[C]. Artificial Intelligence and Statistics. 2015: 562-570.

[9] Gan L, Jensen G J. Electron tomography of cells[J]. Quarterly reviews of biophysics, 2012, 45(1): 27-56.

[10] Allison Doerr. Cryo-electron tomography. Nature Methods, volume 14, page 34; December, 2016

[11] Oikonomou C M, Chang Y W, Jensen G J. A new view into prokaryotic cell biology from electron cryotomography[J]. Nature Reviews Microbiology, 2016, 14(4): 205.

[12] Vanhecke D1, Asano S, et al. Cryo-electron tomography: methodology, developments and biological applications, 2011 Jun;242(3):221-7.

[13] Rossitza N. Irobalieva, Bruno Martins, Ohad Medalia. Cellular structural biology as revealed by cryo-electron tomography, J Cell Sci 2016 129: 469-476

[14] Lui V, Rigort A, Baumeister W. Cryo-electron tomography: the challenge of doing structural biology in situ[J]. J Cell Biol, 2013, 202(3): 407-419.

[15] Asano S, Fukuda Y, Beck F, et al. A molecular census of 26S proteasomes in intact neurons[J]. Science, 2015, 347(6220): 439-442.

[16] Koning RI1, Koster AJ2, Sharp TH3. Advances in cryo-electron tomography for biology and medicine. 2018, May;217:82-96.

[17] Murata K, Liu X, Danev R, et al. Zernike phase contrast cryo-electron microscopy and tomography for structure determination at nanometer and subnanometer resolutions[J]. Structure, 2010, 18(8): 903-912.

[18] Zhao G, Zhou B, Wang K, Jiang R, Xu M. Respond-CAM: Analyzing Deep Models for 3D Imaging Data by Visualizations. Medical Image Computing and Computer Assisted Intervention (MICCAI) 2018. arXiv: 1806.00102

[19] Rigort A, Buerlein F J B, Villa E, et al. Focused ion beam micromachining of eukaryotic cells for cryoelectron tomography[J]. Proceedings of the National Academy of Sciences, 2012.

[20] Liu C, Zeng X, Lin R, Liang X, Freyberg Z, Xing E, Xu M. Deep learning based supervised semantic segmentation of Electron CryoSubtomograms. IEEE International Conference on Image Processing (ICIP) 2018. arXiv:1802.04087

[21] Ekin D. Cubuk, Barret Zoph, et al. Intriguing Properties of Adversarial Examples. arXiv preprint, arXiv:1711.02846

[22] Haixiang G, Yijing L, Shang J, et al. Learning from class-imbalanced data: Review of methods and applications[J]. Expert Systems with Applications, 2017, 73: 220-239.

[23] Levi G, Hassner T. Age and gender classification using convolutional neural networks[C]. Proceedings of the IEEE Conference on Computer Vision and Pattern Recognition Workshops. 2015: 34-42.

[24] Janowczyk A, Madabhushi A. Deep learning for digital pathology image analysis: A comprehensive tutorial with selected use cases[J]. Journal of pathology informatics, 2016, 7.

[25] Jaccard N, Rogers T W, Morton E J, et al. Detection of concealed cars in complex cargo X-ray imagery using deep learning[J]. Journal of X-ray Science and Technology, 2017, 25(3): 323-339.

[26] Wang K J, Makond B, Chen K H, et al. A hybrid classifier combining SMOTE with PSO to estimate 5-year survivability of breast cancer patients[J]. Applied Soft Computing, 2014, 20: 15-24.

[27] Chawla N V, Bowyer K W, Hall L O, et al. SMOTE: synthetic minority over-sampling technique $[\mathrm{J}]$. Journal of artificial intelligence research, 2002, 16: 321-357.

[28] Christian Szegedy, Wei Liu, et al. Going deeper with convolutions CVPR, 2015.

[29] Arpit, S. Jastrzebski, Y. Bengio, et al. A closer look at memorization in deep networks. ICML, 2017.

[30] Shen L, Lin Z, Huang Q. Relay backpropagation for effective learning of deep convolutional neural networks[C]. European conference on computer vision. Springer, Cham, 2016: 467-482.

[31] Mikel Galar, Alberto Fernandez, et,al. A Review on Ensembles for the Class Imbalance Problem: Bagging-, Boosting-, and Hybrid-Based Approaches, 2011, IEEE TRANSACTIONS ON SYSTEMS, MAN, AND CYBERNETICSPART C: APPLICATIONS AND REVIEWS

[32] Kubat M, Matwin S. Addressing the curse of imbalanced training sets: one-sided selection[C]. ICML. 1997, 97: 179-186.
[33] Barandela R, Rangel E, Snchez J S, et al. Restricted decontamination for the imbalanced training sample problem[C]. Iberoamerican Congress on Pattern Recognition. Springer, Berlin, Heidelberg, 2003: 424-431.

[34] Eggermont J, Kok J N, Kosters W A. Genetic programming for data classification: Partitioning the search space[C]. Proceedings of the 2004 ACM symposium on Applied computing. ACM, 2004: 1001-1005.

[35] Abraham J. Wyner, Matthew Olson, et,al. Explaining the Success of AdaBoost and Random Forests as Interpolating Classifiers. arXiv preprint arXiv:1504.07676 2017.

[36] Qing-Yan Yin, Jiang-She Zhang, et, al. An Empirical Study on the Performance of Cost-Sensitive Boosting Algorithms with Different Levels of Class Imbalance. Volume 2013, Article ID 761814, 12 pages.

[37] T. N. Sainath, O. Vinyals, A. Senior, and H. Sak, Convolu- tional, long short-term memory, fully connected deep neural networks, in IEEE International Conference on Acoustics, Speech and Signal Processing (ICASSP). IEEE, 2015, pp. 45804584.

[38] D.Palaz,R.Collobertetal.,Analysis of cnn-based speechrecog- nition system using raw speech as input, in Proceedings of Interspeech, 2015.

[39] Tang Y, Zhang Y Q, Chawla N V, et al. SVMs modeling for highly imbalanced classification[J]. IEEE Transactions on Systems, Man, and Cybernetics, Part B (Cybernetics), 2009, 39(1): 281-288.

[40] Szegedy C, Ioffe S, Vanhoucke V, et al. Inception-v4, inception-resnet and the impact of residual connections on learning[C]. AAAI. 2017, 4: 12

[41] Urvesh Bhowanm, Mark Johnston, et al. Reusing Genetic Programming for Ensemble Selection in Classification of Unbalanced Data. IEEE Transactions on Evolutionary Computation, Volume: 18 Issue: 6, 2014

[42] Galar M, Fernandez A, Barrenechea E, et al. A review on ensembles for the class imbalance problem: bagging-, boosting-, and hybrid-based approaches[J]. IEEE Transactions on Systems, Man, and Cybernetics, Part C (Applications and Reviews), 2012, 42(4): 463-484.

[43] T. DeVries and G. W. Taylor. Dataset augmentation in feature space. ICLR Workshops, 2017.

[44] Behzad Hasani, et al. Facial Expression Recognition Using Enhanced Deep 3D Convolutional Neural Networks, IEEE Conference on Computer Vision and Pattern Recognition Workshops, 2017

[45] Christian Szegedy, Vincent Vanhoucke, et al. Rethinking the Inception Architecture for Computer Vision, CVPR, 2016.

[46] Mayuri S. Shelke, et al. A Review on Imbalanced Data Handling Using Undersampling and Oversampling Technique, IJRTER, Volume 03, Issue 04; April, 2017

[47] Mateusz Buda, Atsuto Maki, et al. A systematic study of the class imbalance problem in convolutional neural networks, arXiv preprint arXiv:1710.05381, 2017

[48] Zhang H, Cisse M, Dauphin Y N, et al. mixup: Beyond empirical risk minimization[J]. arXiv preprint arXiv:1710.09412. 2017.

[49] Lin T Y, Goyal P, Girshick R, et al. Focal loss for dense object detection $[\mathrm{J}]$. IEEE transactions on pattern analysis and machine intelligence, 2018.

[50] Guo Q, Lehmer C, Martnez-Snchez A, Rudack T, Beck F, Hartmann H, Prez-Berlanga M, Frottin F, Hipp MS, Hartl FU, Edbauer D. In situ structure of neuronal C9orf72 Poly-GA aggregates reveals proteasome recruitment. Cell. 2018 Feb 8;172(4):696-705. 